\title{
APPLIED MAGNETOOPTIC SOLITON DYNAMICS: TM AND TE-TM-DRIVEN SYSTEMS
}

\author{
A.D. Boardman ${ }^{*}$, K. XIE and M. XIE \\ Photonics and Nonlinear Science Group, Joule Physics Laboratory \\ School of Sciences, University of Salford, Salford, M5 4WT, U.K.
}

\begin{abstract}
Ideas concerning the use of magnetooptic materials to control spatial solitons are introduced. It is shown that control of spatial solitons can be achieved through the use of TM modes TE-TM interactions. Potential functions can be defined through a Lagrangian analysis and optimisation is possible to facilitate energy transfer between TE and TM waves.
\end{abstract}

PACS numbers: 42.65.Tg

\section{Introduction}

Polarisation-coupled spatial solitons [1-3] in optical planar waveguides have been investigated for some time now using Whitham's average variational principle [4]. It is encouraging that analytical forms can be found this way and that numerical work usually confirms that the true solitons agree with the mathematical analysis. It is safe to say, then, that significant mathematical progress has been made in many cases and have been verified by accurate (exact) numerical simulation. The variational principle gives the averaged equations for all the slowly varying functions. A detailed justification of it has been given by Whitham [4]. He points out that the variational approach is not a separate method, but includes the usual expansion approach and, in so doing, permits the generation of quite general results. It is quite capable of dealing with stability questions but, naturally, the accuracy of any description of spatial soliton beam dynamics must depend upon the choice of trial function. There is plenty of evidence to suggest, however, that a good and general choice leads to credible conclusions in which great confidence can be placed.

Thin garnet films have been of interest for decades now. In fact, a whole range of microwave signal processing devices [5-8] such as filters, correlators, spectrum analysers, switches, modulators, frequency shifters, and tunable filters are either in use or appear to be on the horizon. Such devices will be even more useful if power can be added in as another degree of flexibility. In other words, the study

* corresponding author; e-mail: a.d.boardman@physics.salford.ac.uk 
of nonlinear magnetooptical interactions is of prime importance. This is a realistic prospect because modern film production technology is so much better than it used to be, that what is possible today, literally, bears no comparison with what was possible in previous decades. It is now easy to make the fundamental integrated optical building block, which is the channel waveguide. It is also possible to imagine future integration of magnetooptic devices with semiconducting substrates containing active devices, like lasers, detectors, and amplifiers.

The two orientations of the magnetisation $\boldsymbol{M}$, to the propagation direction of a wave in a slab of magnetooptic material to be discussed here are longitudinal or transverse, in the tensor form

$$
\begin{aligned}
\text { longitudinal } & \varepsilon=\left(\begin{array}{ccc}
n^{2} & -\mathrm{i} Q n^{2} & 0 \\
\mathrm{i} Q n^{2} & n^{2} & 0 \\
0 & 0 & n^{2}
\end{array}\right), \\
\text { transverse } & \varepsilon=\left(\begin{array}{ccc}
n^{2} & 0 & 0 \\
0 & n^{2} & -\mathrm{i} Q n^{2} \\
0 & \mathrm{i} Q n^{2} & n^{2}
\end{array}\right),
\end{aligned}
$$

$n$ is the refractive index, $Q$ is called the magnetooptical parameter and, for practical reasons, $n$ is = assumed to be the same in each diagonal term. This is a realistic prospect because it would appear that there has never been a better time to pursue the combination of nonlinearity and magnetooptics.

\section{Basic theory}

For guided wave propagation along the z-axis, the electric field vector is $\boldsymbol{E}=\left[E_{x}, E_{y}, E_{z}\right], E_{z} \cong 0$, so the longitudinal configuration couple $E_{x}$ and $E_{y}$ as opposed to coupling $E_{y}$ to $E_{z}$ (transverse). Maxwell's equations, for an angular frequency $\omega$, reduce to

$$
\begin{aligned}
& \nabla \cdot(\varepsilon \cdot \boldsymbol{E})=0, \\
& \nabla^{2} \boldsymbol{E}-\nabla(\nabla \cdot \boldsymbol{E})+\frac{\omega^{2}}{c^{2}} \varepsilon \cdot \boldsymbol{E}=0,
\end{aligned}
$$

where $c$ is the velocity of light in a vacuum. Considering only the magnetooptics, for the time being, the longitudinal case gives

$$
\begin{aligned}
& \nabla^{2} E_{x}+\frac{\omega^{2}}{c^{2}} n^{2} E_{x}-\mathrm{i} Q\left(\frac{\partial^{2} E_{y}}{\partial x^{2}}-\frac{\partial^{2} E_{x}}{\partial x \partial y}\right)-\mathrm{i} Q n^{2} \frac{\omega^{2}}{c^{2}} E_{y}=0 \\
& \nabla^{2} E_{y}+\frac{\omega^{2}}{c^{2}} n^{2} E_{y}-\mathrm{i} Q\left(\frac{\partial^{2} E_{y}}{\partial x \partial y}-\frac{\partial^{2} E_{x}}{\partial y^{2}}\right)+\mathrm{i} Q n^{2} \frac{\omega^{2}}{c^{2}} E_{x}=0
\end{aligned}
$$

Clearly the term involving $Q$ is a perturbation and, furthermore, $x, y$, and $z$ are best measured, in units of $c / \omega$. Hence,

$$
\nabla^{2} E_{j}+\left(n^{2}+\Delta n_{j}^{2}\right) E_{j}=0, \quad j=x, y,
$$

in which

$$
\Delta n_{x}^{2}=-\mathrm{i} Q\left[\frac{1}{E_{x}} \frac{\partial^{2} E_{y}}{\partial x^{2}}-\frac{1}{E_{x}} \frac{\partial^{2} E_{x}}{\partial x \partial y}+n^{2} \frac{E_{y}}{E_{x}}\right],
$$




$$
\Delta n_{y}^{2}=\mathrm{i} Q\left[\frac{1}{E_{y}} \frac{\partial^{2} E_{x}}{\partial y^{2}}-\frac{1}{E_{y}} \frac{\partial^{2} E_{y}}{\partial x \partial y}+n^{2} \frac{E_{x}}{E_{y}}\right]
$$

are the magnetooptic perturbations, referred to earlier

The solution technique is to referred to earlier. First of all imagine that propagation, along $z$, in a planar waveguide, takes place, with the $y$-axis being set perpendicular to the plane of the guide, and then to factor out the transverse $(y)$ dependence by writing [3]

$$
E_{j}(x, y, z)=\Gamma A_{j}(y) B_{j}(x, z) \exp \left(\mathrm{i} \frac{\omega}{c} \beta z\right),
$$

where $\Gamma$ is a normalisation constant, the transverse guided wave profiles, $A_{j}(y)$, are assumed to be unaffected by the perturbation, $B_{j}(x, z)$ are slowly varying amplitudes, $(\omega / c) \beta$ is a common propagation wave number and, finally, $\beta$ is an average of the unperturbed wave numbers $\beta_{x}$ and $\beta_{y}$. The substitution requires the introduction of common separation constants $\bar{\beta}_{x}$ and $\bar{\beta}_{y}$, so that the equations for $A_{j}$ and $B_{j}$, after the transformation $z / 2 \beta \rightarrow z$, are

$$
\begin{aligned}
& \frac{\partial^{2} A_{j}}{\partial y^{2}}+\left(n^{2}+\Delta n_{j}^{2}-\bar{\beta}_{j}^{2}\right) A_{j}=0, \\
& \mathrm{i} \frac{\partial B_{j}}{\partial z}+\frac{\partial^{2} B_{j}}{\partial x^{2}}+\left(\bar{\beta}_{j}^{2}-\beta^{2}\right) B_{j}=0,
\end{aligned}
$$

where $\beta^{2} \cong \beta_{x}^{2}-\beta\left(\beta_{x}-\beta_{y}\right)$ and $\bar{\beta}_{j}^{2}-\beta_{j}^{2}$ are shifts in the propagation constants, created by the perturbation. Also $\beta_{x} \cong \beta_{y}$ so that $\beta^{2} \cong \beta_{x}^{2}$.

Since most of the terms, in the equations for $B_{x}$ and $B_{y}$, can be neglected, because the system is weakly guiding, the coupled equations that describe magnetooptic interaction reduce to

$$
\begin{aligned}
& \mathrm{i} \frac{\partial B_{x}}{\partial z}+\frac{\partial^{2} B_{x}}{\partial x^{2}}-\mathrm{i} Q_{1} B_{y}=0, \\
& \mathrm{i} \frac{\partial B_{y}}{\partial z}+\frac{\partial^{2} B_{y}}{\partial x^{2}}+\mathrm{i} Q_{1} B_{x}=0,
\end{aligned}
$$

where $Q_{1}=2 \int n^{2} Q A_{x} A_{y} \mathrm{~d} y /\left(\int A_{x}^{2} \mathrm{~d} y+\int A_{y}^{2} \mathrm{~d} y\right)$.

\section{Dynamics of solitons}

The nonlinearity will now be introduced by assuming that they are third-order nonlinear $\left[\xi^{(3)}\right]$ materials, which have nonlinear refractive index changes

$$
\Delta n_{j}^{2}=\alpha\left[\left(\left|E_{j}\right|^{2}+\left|E_{k}\right|^{2}\right)+f\left(\frac{E_{j}^{*}}{E_{j}} E_{k}^{2}-\left|E_{k}\right|^{2}\right)\right]
$$

where $k, j=x, y, j \neq k$ and $f=3 \chi_{y x y x}^{(3)} / 4 \alpha$, and $\chi_{y x y x}^{(3)}$ is a component of a fourth-rank tensor $\chi_{i j k l}^{(3)}$ that characterises third-order nonlinear optical susceptibility $[9,10]$. 
The combined effects of optical nonlinearity and magnetooptic influence are presented by the following coupled equations:

$\mathrm{i} \frac{\partial \psi_{x}}{\partial z}+\frac{\partial^{2} \psi_{x}}{\partial x^{2}}+2\left[\left|\psi_{x}\right|^{2}+\left|\psi_{y}\right|^{2}\right] \psi_{x}+2 f\left(\psi_{x}^{*} \psi_{y}-\psi_{x} \psi_{y}^{*}\right) \psi_{y}-\mathrm{i} Q \psi_{y}=0$

$\mathrm{i} \frac{\partial \psi_{y}}{\partial z}+\frac{\partial^{2} \psi_{y}}{\partial x^{2}}+2\left[\left|\psi_{x}\right|^{2}+\left|\psi_{y}\right|^{2}\right] \psi_{y}+2 f\left(\psi_{y}^{*} \psi_{x}-\psi_{y} \psi_{x}^{*}\right) \psi_{x}+\mathrm{i} Q \psi_{x}=0$

where $\sqrt{\frac{\alpha^{\prime}}{2}} B_{j} \rightarrow \psi_{j}, \alpha^{\prime}=\alpha \int\left|A_{y}\right|^{4} \mathrm{~d} y /\left(\int\left|A_{y}\right|^{2} \mathrm{~d} y\right)^{2}$ and $Q_{1}$ is redefined to be $Q$, once again, for convenience.

\section{Counter-rotating polarisations}

These are defined as $\psi_{+}=\frac{1}{\sqrt{2}}\left(\psi_{x}+\mathrm{i} \psi_{y}\right)$ and $\psi_{-}=\frac{1}{\sqrt{2}}\left(\psi_{x}-i \psi_{y}\right)$ so that, together with the definitions $\mu=(1+f) /(1-f), \sqrt{1-f} \psi_{+}=\psi_{1}$ and $\sqrt{1-f} \psi_{-}=\psi_{2}$ the following coupled equations must emerge:

$$
\begin{aligned}
& i \frac{\partial \psi_{1}}{\partial z}+\frac{\partial^{2} \psi_{1}}{\partial x^{2}}+2\left[\left|\psi_{1}\right|^{2}+\mu\left|\psi_{2}\right|^{2}\right] \psi_{1}-Q \psi_{1}=0, \\
& i \frac{\partial \psi_{2}}{\partial z}+\frac{\partial^{2} \psi_{2}}{\partial x^{2}}+2\left[\left|\psi_{2}\right|^{2}+\mu\left|\psi_{1}\right|^{2}\right] \psi_{2}+Q \psi_{2}=0 .
\end{aligned}
$$

The Lagrangian is

$$
\begin{aligned}
L= & \sum_{j=1}^{2}\left[\frac{\mathrm{i}}{2}\left(\psi_{j}^{*} \frac{\partial \psi_{j}}{\partial z}-\frac{\partial \psi_{j}^{*}}{\partial z} \psi_{j}\right)-\left|\frac{\partial \psi_{j}}{\partial x}\right|^{2}+\left|\psi_{j}\right|^{4}\right] \\
& +2 \mu\left|\psi_{1}\right|^{2}\left|\psi_{2}\right|^{2}-Q\left|\psi_{1}\right|^{2}+Q\left|\psi_{2}\right|^{2}
\end{aligned}
$$

and good trial function selections, based upon the uncoupled solitons, are

$$
\psi_{j}=\eta_{j} \operatorname{sech}\left[\eta_{j}\left(x-x_{j}\right)\right] \exp \left[\mathrm{i} \frac{\xi_{j}}{2}\left(x-x_{j}\right)+\mathrm{i} \phi_{j}\right]
$$

and the average Lagrangian [4], is obtained by integrating over all $x$. The outcome, when used in Euler's equations yields the evolution dynamics equations using $Q=Q(x)$

$$
\begin{gathered}
\frac{\mathrm{d} \eta_{j}}{\mathrm{~d} z}=0, \quad \frac{\mathrm{d} x_{j}}{\mathrm{~d} x}=\xi \\
\frac{\eta_{1}}{2} \xi_{1}^{2}+\frac{\eta_{2}}{2} \xi_{2}^{2}-2 \mu \eta_{1}^{2} \eta_{2}^{2} \int \operatorname{sech}^{2}\left[\eta_{1}\left(x-x_{1}\right)\right] \operatorname{sech}^{2}\left[\eta_{2}\left(x-x_{2}\right)\right] \mathrm{d} x \\
+\int Q(n) \eta_{1}^{2} \operatorname{sech}^{2}\left[\eta_{1}\left(x-x_{1}\right)\right] \mathrm{d} x-\int Q(n) \eta_{2}^{2} \operatorname{sech}^{2}\left[\eta_{2}\left(x-x_{2}\right)\right] \mathrm{d} x=2 H,
\end{gathered}
$$

where $2 H$ is just a constant.

Now suppose that the evolution equations seek to quantify the slow variations of the trial function parameters, when a particular question is posed. For example, suppose that two interacting solitons have the properties

$$
\eta_{1}=\eta_{2}=1, \quad \xi_{2}=-\xi_{1}=\xi, \quad x_{2}=-x_{1}=\Delta
$$


and that the magnetisation distribution is [3]

$$
Q(x)=\left\{\begin{array}{cc}
-Q_{0} & x<0 \\
Q_{0} & x>0
\end{array}\right.
$$

then

$$
H=\frac{\xi^{2}}{2}-4 \mu\left[\frac{1}{\tanh ^{2}(2 \Delta)}-1\right]\left[\frac{2 \Delta}{\tanh (2 \Delta)}-1\right]-2 Q_{0} \tanh (\Delta)
$$

where $H$ can now be viewed as the Hamiltonian energy. This equation implies that a potential energy $U(\Delta)$ exists between the interacting polarised solitons, which is

$$
U(\Delta)=-4 \mu\left[\frac{1}{\tanh ^{2}(2 \Delta)}-1\right]\left[\frac{2 \Delta}{\tanh (2 \Delta)}-1\right]-2 Q_{0} \tanh (\Delta)
$$

In fact, more generally, [3]

$$
\begin{aligned}
U(\Delta) & =-4 \mu\left[\frac{1}{\tanh ^{2}(2 \Delta)}-1\right]\left[\frac{2 \Delta}{\tanh (2 \Delta)}-1\right]+\frac{1}{2} \int Q(x) \operatorname{sech}^{2}(x+\Delta) \mathrm{d} x \\
& -\frac{1}{2} \int Q(x) \operatorname{sech}^{2}(x-\Delta) \mathrm{d} x
\end{aligned}
$$

Figure 1 shows a possible magnetooptic waveguide, which consists of a nonlinear waveguide and an interesting magnetooptic cladding. In this cladding, the saturation magnetic fields $\boldsymbol{H}$ are in opposite directions, giving rise to the distribution $Q(x)$ shown in the figure. The plot of $U(\Delta)$ against $\Delta$ is shown in Fig. 2 for various values of $Q_{0}$. For $Q_{0}=0$ the potential well is symmetric, as expected. For $Q_{0}= \pm 0.2$ symmetric wells result, which gives the possibility of novel control of the soliton dynamics by the magnetic field. In the absence of a magnetic field, Fig. 3 shows that the collapse [3] of the solitons is inevitable, provided that they propagate far enough.
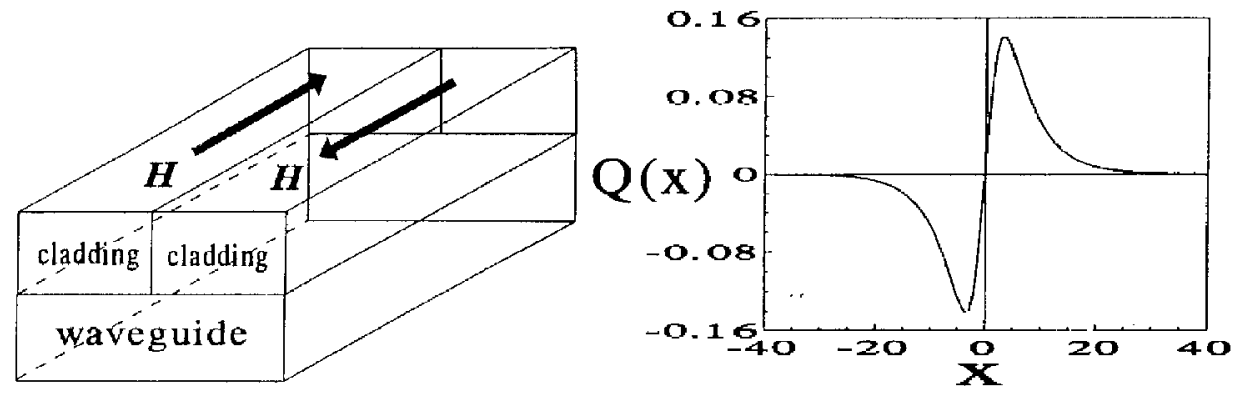

Fig. 1. A longitudinal magnetooptic configuration and a possible $Q(x)$ distribution, $Q(x)=Q_{0} \tanh (0.4 x) / \cosh (0.2 x)$. 


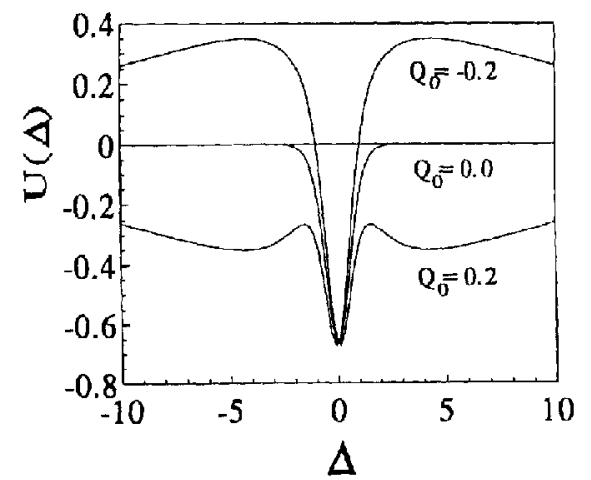

Fig. 2. Potential functions for $Q_{0}=-0.2,0,0.2$.

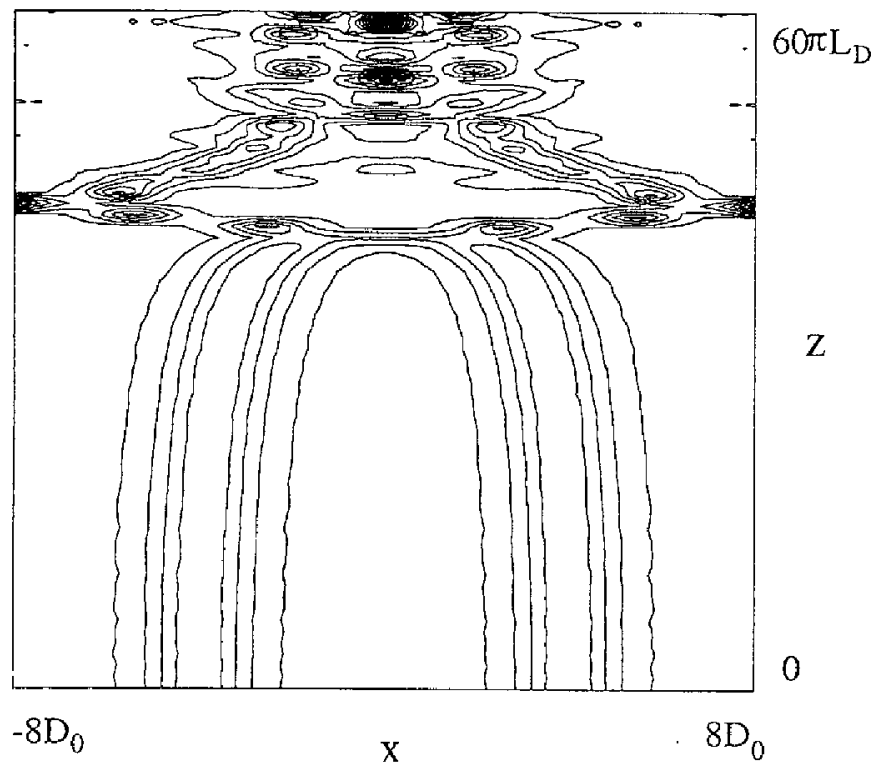

Fig. 3. Propagation, to collision, of two solitary beams, in the absence of an applied magnetic field.

\section{TE-TM waves}

TM or mixed TE-TM waves can be launched in a structure which has a current strip deposited upon it that provides a sufficiently strong magnetic field to saturate the magnetooptic material.

The magnetic field $\boldsymbol{H}$ is created $[11,12]$ by a current $I$ flowing in a stripline along the surface of the device. There is a component of $\boldsymbol{H}$ perpendicular to the 
propagation direction $z$ and the magnetic material, which could be Bi-doped YIG, or a material like lutetium. $I$ is $\sim 60 \mathrm{~mA}$, producing $H \sim 400 \mathrm{Oe}$, which is enough to saturate the magnetisation.

For the arbitrary magnetic field direction, shown in Fig. 4, there are general magnetooptic contributions to the dielectric tensor $\varepsilon$

$$
\varepsilon=n^{2}\left(\begin{array}{ccc}
1 & -\mathrm{i} Q \cos \theta & \mathrm{i} Q \sin \theta \sin \phi \\
\mathrm{i} Q \cos \theta & 1 & -\mathrm{i} Q \sin \theta \cos \phi \\
-\mathrm{i} Q \sin \theta \sin \phi & \mathrm{i} Q \sin \theta \cos \phi & 1
\end{array}\right) .
$$

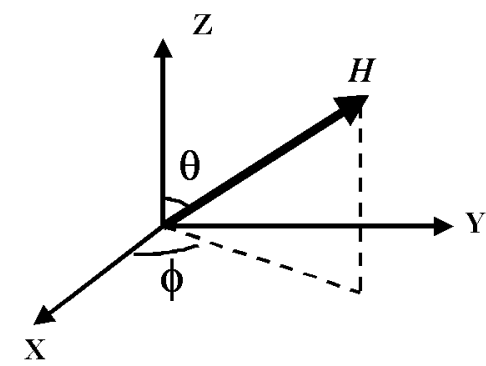

Fig. 4. The coordinate system.

The simplest third-order Kerr nonlinearity couples equations for the amplitudes $A_{1}, A_{2}$, respectively, of the TE and TM waves. After writing the electric field components as

$$
\begin{array}{ll}
\text { TE }: & A_{1} \xi_{x} \exp \left(-\mathrm{i} \frac{\omega}{c} \beta_{\mathrm{TE}} z\right) ; \\
\mathrm{TM}: & A_{2} \xi_{y} \exp \left(-\mathrm{i} \frac{\omega}{c} \beta_{\mathrm{TM}} z\right), \quad A_{2} \xi_{z} \exp \left(-\mathrm{i} \frac{\omega}{c} \beta_{\mathrm{TM}} z\right),
\end{array}
$$

which all have a common factor $\exp (\mathrm{i} \omega t), \omega$ being the angular frequency, parabolic equations for $A_{2}$ can be found. For example,

$$
\begin{aligned}
& 2 \mathrm{i} \frac{c}{\omega} \frac{\partial A_{2}}{\partial z}=\frac{c^{2}}{\omega^{2} \beta_{2}} \frac{\partial^{2} A_{2}}{\partial x^{2}}-\mathrm{i}\left(\bar{\varepsilon}_{x y}-\mathrm{i} \bar{\varepsilon}_{x z}\right) \exp \left(\mathrm{i} \frac{\omega}{c} \gamma z\right) A_{1}+2 \bar{\varepsilon}_{y z} A_{2}-\mathrm{i} \bar{\alpha} A_{2} \\
& \quad+\bar{\chi}\left(A_{1}\left|A_{1}\right|^{2}+A_{2}\left|A_{2}\right|^{2}\right) .
\end{aligned}
$$

There is a similar equation for $A_{1} \cdot \bar{\varepsilon}_{i j}$ are now the elements of the magnetooptic tensor, $\bar{\alpha}$ is an average absorption coefficient and $\bar{\chi}$ is an average nonlinear susceptibility. Indeed, all these quantities are now averaged over the selected waveguide.

The phase mismatch between the TE and TM waves is $\gamma=\beta_{\mathrm{TE}}-\beta_{\mathrm{TM}}$, where $\beta_{\mathrm{TE}}$ and $\beta_{\mathrm{TM}}$ are the effective indices of the TE or TM waves. For TE-TM coupling there is clearly a need to deal with the $\exp [\mathrm{i}(\omega / c) \gamma z]$ factor, to prevent it from averaging to zero as propagation proceeds along $z$. Hence the structure needs to be optimised, if a magnetooptic influence is to be apparent in this application. If $\bar{\varepsilon}_{x y}=0, \bar{\varepsilon}_{x z}=0$, leaving only $\bar{\varepsilon}_{y z}$, no phase problem exists. In fact, if $\bar{\varepsilon}_{y z}$ is also a constant it introduces only a simple phase shift. Here, however, $\bar{\varepsilon}_{y z}=\bar{\varepsilon}_{y z}(x)$ and this creates a current-driven potential well that may capture a soliton. The ideal case, in which the current line is infinitely thin, is very instructive but the 
consequences of using a finite width stripline can easily be found. For a soliton beam width $D_{0}$, it is a parameter $\nu=2 \beta_{\mathrm{TM}}\left(\omega^{2} / c^{2}\right) D_{0}^{2} \bar{\varepsilon}_{y z}$ that really determines the well characteristics and, again, a simple Lagrangian analysis reveals all the presence of the well. The analysis yields, this time, the simple well shape

$$
U\left(x_{0}\right)=\nu_{0}\left[\tanh \left(\frac{p}{2}-x_{0}\right)+\tanh \left(\frac{p}{2}+x_{0}\right)\right],
$$

where $\nu$ is now (realistically) approximated to $\nu=0(x>p / 2$ or $<-p / 2)$ and $\nu=-\nu_{0}$ for $-p / 2<x<p / 2 . p$ is a measure of the well width determined from a calculation of the magnetic field distribution. $x_{0}$ is the initial position of the soliton on the $x$-axis. The application is beautifully simple but powerful. The current $I$ creates an $x$-dependent magnetic field distribution and a potential barrier, or well, is created depending upon the current flow direction or soliton propagation direction. Solitons with large angles of incidence to the barrier, and a small kinetic energy, are reflected. The total power is $\left(c / \omega D_{0}\right)^{2}\left(4 D_{0} / \beta_{2} \bar{\chi}\right)$ so both power and the magnetooptic parameter change as $D_{0}$ changes: $D_{0} \uparrow \Rightarrow \nu \downarrow, p \downarrow$, and $D_{0} \downarrow \Rightarrow \nu \downarrow, p \uparrow$. The effects can be seen in Fig. 5. Also, the sketch in Fig. 6 shows that the soliton can now be routed to make a soliton circulator.
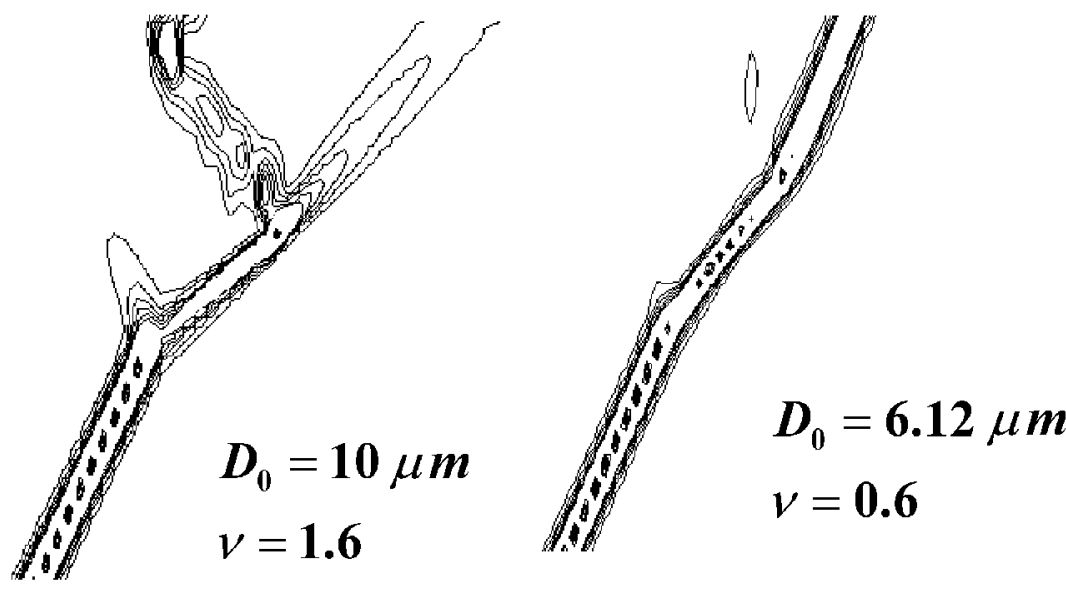

Fig. 5. TM solitons in current-created well.

For the longitudinal case, the basic parabolic equation transforms to

$$
\gamma \psi_{1}+2 \mathrm{i} \frac{c}{\omega} \frac{\partial \psi_{1}}{\partial z}=\frac{c^{2}}{\omega^{2} \beta_{0}} \frac{\partial^{2} \psi_{1}}{d z^{2}}+\mathrm{i} \bar{\varepsilon}_{x y} \psi_{2}+\bar{\chi}\left[\psi_{1}\left|\psi_{2}\right|^{2}+\psi_{1}\left|\psi_{2}\right|^{2}\right] .
$$

This is the generalisation to $\gamma \neq 0$, where $\psi_{1,2}$ is related to $A_{1,2}$ in such a way as to bring the phase factor, $\gamma$ onto the line of the equation.

If the phase mismatch is $\gamma \gg \bar{\varepsilon}_{x y}$ then magnetooptic effects will be suppressed, so it is important to be able to optimise the structure. This optimisation, can use a refractive index variation through $\mathrm{Bi}$-doping of iron garnet layers but it must also vary the quantities averaged over the guiding structure by changing the guide thickness. 


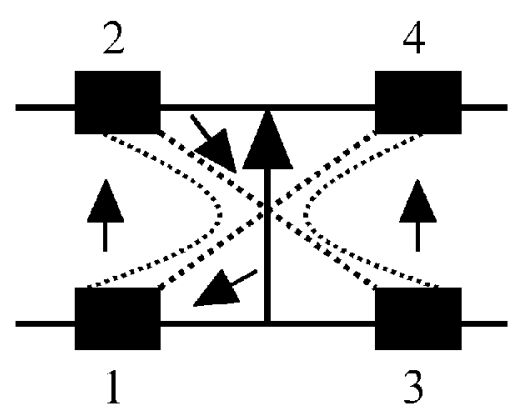

Fig. 6. Soliton circulator.

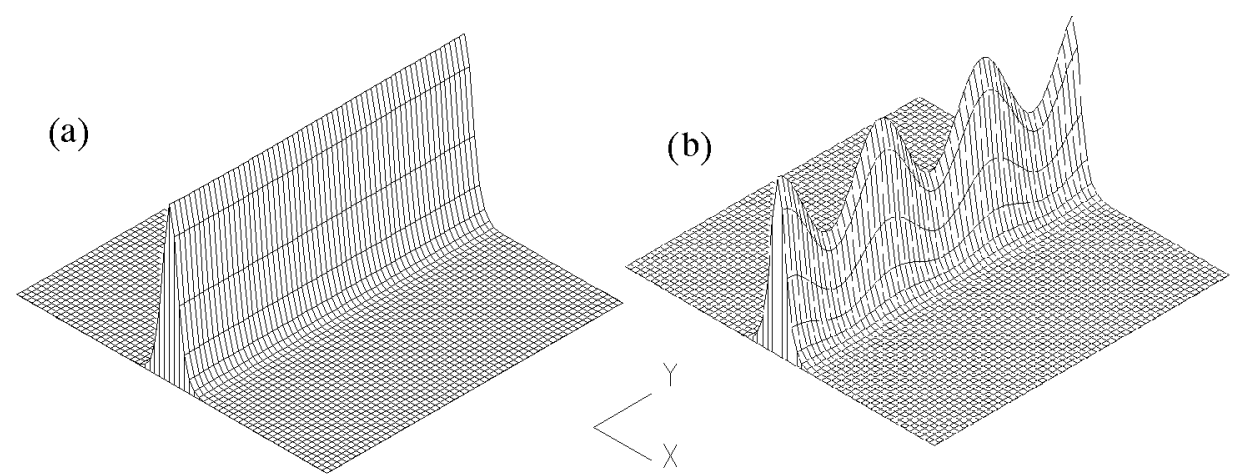

Fig. 7. Pre-optimisation TE soliton (a), optimised structure showing TE-TM coupling of solitons in a nonlinear layer sitting on a magnetooptic substrate (b).

These actions will achieve both a reduction in $\gamma$ and remarkable enhancements of both the magnetooptical and the nonlinear effects. Figure 7 shows a TE polarised spatial soliton in a planar waveguide structure (like AlGaAs) before, and after, enhancement and it is clear that energy transfer from TE to the TM wave (and vice versa) is achieved. $D_{0}$ is optimised to $1.3 \mu \mathrm{m}$ and $\mathrm{Lu}_{3-x} \mathrm{Bi}_{x} \mathrm{Fe}_{5-y} \mathrm{Ga}_{y} \mathrm{O}_{12}$ is used at $x=1.4, y=1.5$. The diffraction length is of the order of magnitude of millimeters, so absorption is not a problem. Power levels are a function of the nonlinear material and in this application are $\sim 100 \mathrm{~W}$.

\section{References}

[1] A.D. Boardman, K. Xie, Phys. Rev. Lett. 76, 4591 (1995).

[2] A.D. Boardman, K. Xie, Phys. Rev. E 55, 1899 (1997).

[3] A.D. Boardman, K. Xie, J. Opt. Soc. Am. B 15, 3102 (1997).

[4] G.B. Whitham, Linear and Nonlinear Waves, John Wiley \& Sons, New York 1974.

[5] D.S. Stancil, IEEE J. Quantum Electron. 27, 61 (1991).

[6] U. Pustogowa, W. Hubner, K.H. Benneman, Phys. Rev. B 49, 10031 (1994). 
[7] M. Wallenhorst, M. Niemoller, H. Dotsch, P. Hertel, R. Gerhardt, B. Gather, J. App. Phys. 77, 2902 (1995).

[8] A.K. Zvezdin, V.A. Kotov, Modern Magnetooptics and Magnetooptical Materials, IoP, Bristol 1997.

[9] A.D. Boardman, T. Twardowski, Phys. Rev. A 39, 2481 (1989).

[10] A.D. Boardman, K. Xie, A.A. Zharov, Phys. Rev. A 51, 692 (1995).

[11] A.D. Boardman, M. Xie, Phys. Rev. E., to be published.

[12] A.D. Boardman, M. Xie, in: Complex Mediums, Ed. A. Lakhtakia, Proc. SPIE 4097, 59 (2000). 